\title{
CHAPITRE 2
}

\section{La responsabilité \\ de la société-mère}

pour la faute de la filiale

\author{
Wojciech Popiołek \\ Professeur à l'Université de Silésie
}

\section{Introduction}

La règle d'autonomie juridique des sociétés de capitaux - résultant de la conception de l'essence de la personnalité juridique adoptée en droit polonais - est considérée comme l'une des règles fondamentales du droit des sociétés commerciales. Elle se manifeste par l'autonomie juridique réciproque de la société de capitaux et de ses associés. Il en est de même en ce qui concerne les sociétés fonctionnant dans les groupes de sociétés, où la société dominante (société-mère) est un associé dominant de la société dépendante (filiale). Le rapport de domination ne conduit pas à la suppression de la règle d'autonomie de la société et de son associé.

De la règle d'autonomie résulte que les associés ne sont pas responsables des obligations de la société, celle-ci n'étant pas responsable des obligations des associés (l'art. $151 \$ 4$ et l'art. $301 \$ 5$ du Code des sociétés commerciales $^{1}$ ). Le patrimoine de la société sert à satisfaire les prétentions de ses créanciers, ces derniers ne pouvant pourtant pas demander la satisfaction de leurs prétentions aux associés de la société quelle que soit leur influence exercée sur le fonctionnement de la société.

Cette conséquence découlant de la règle de l'autonomie réciproque des sociétés et de leurs associés est pourtant en contradiction manifeste avec la réalité économique, dans laquelle les groupes de sociétés liées par des rapports de domination/dépendance fonctionnent comme dans un organisme économique homogène. Dans les rapports relatifs au

1 Loi du 15 septembre 200 - Code des sociétés commerciales, J. des L. n94, texte 1037 avec mod. («c. s. c. »). 
commerce la société dépendante devient souvent un outil pour son associé dominant - à savoir la société-mère. La société dépendante exerce dans le commerce une activité en son propre nom mais dans l'intérêt économique de la société dominante ou bien de celui d'un groupe de sociétés. Il en résulte la création d'obligations de la filiale à l'égard des tiers, y compris de celles résultant d'un dommage causé à ces derniers. La société dominante bénéficie d'un privilège d'absence de responsabilité bien qu'elle soit à l'origine des conséquences défavorables aux cocontractants de la société dépendante.

La question donc se pose : le fait que la société dominante exerce un contrôle sur sa filiale peut-il justifier l'imputation à la société dominante de la responsabilité pour les obligations de la société dépendante ou la construction de sa responsabilité pour les dommages à l'égard des personnes ayant subi un dommage causé, c'est vrai, directement par les agissements de la filiale - mais ceux-ci ayant été causés par la société-mère.

La problématique de l'affaiblissement de la règle d'autonomie opéré à l'aide de différentes constructions dénommées dans la doctrine étrangère « le percement du voile corporatif» ("piercing the corporate veil»), "l'omission (ignorance) de l'autonomie juridique " ( disregarding corporate identity»), "l'irruption à travers la barrière » ("Durchgriff») ou bien "la gérance de fait » ont également suscité l'intérêt de la doctrine polonaise ${ }^{2}$. Ces constructions visent également à créer la possibilité d'imputer à la société dominante la responsabilité pour les obligations de la société dépendante à l'égard de ses créanciers, y compris pour un dommage causé à un tiers. La réalité socio-économique et ses besoins actuels justifient la recherche de solutions assurant la protection des personnes ayant ainsi subi un dommage, vu - dans le contexte de la dynamique des rapports économiques - le risque croissant de devenir « victime».

La législation polonaise ne prévoit aucun instrument distinct susceptible - et ceci aux fins définies ci-dessus - d'affaiblir l'autonomie des membres d'un groupe de sociétés, indépendamment du degré dans lequel en pratique les agissements de la société dépendante et ses résultats coïncident avec l'objet du groupe, défini par la société dominante. La législation polonaise manque en particulier pratiquement aussi bien de régulation générale de la conséquence de l'existence du rapport de domination que de cadres juridiques clairement définis applicables au fonctionnement

2 Cf. p. ex. A. Opalski, Prawo zgrupowań spółek (Le droit de groupement de sociétés), Varsovie 2012, p. 473 et suiv.; S. Sołtysiński (dans :) S. Sołtysiński, A. Szajkowski, A. Szumański, J. Szwaja, Kodeks spółek handlowych, Komentarz (Code des sociétés commerciales, Commentaire), Varsovie 2008, vol. III, p. 22 et suiv.; S. Włodyka, (dans:) System prawa handlowego (Régime du droit commercial). Vol. 2, Prawo spółek handlowych (Droit des sociétés commerciales), Varsovie 2012, p. 1562 et suiv. 
des contrats de holding ${ }^{3}$. Ces cadres permettraient - en suivant l'exemple de certains législateurs - de créer également les fondements de l'élargissement de l'étendue de responsabilité de la société dominante ${ }^{4}$. On ne peut pas nier le fait que les dispositions de l'article 7 du c.s.c. s'expriment sur certains types des contrats de holding conclus entre la société dominante et les sociétés dépendantes, ces dispositions ne se limitant pourtant qu'à obliger la société à divulguer (et ceci en annexant le document requis au dossier d'enregistrement) des informations précises relatives au contrat de holding. Il s'agit d'informations précisant si (où, le cas échéant - non) le contrat contient les stipulations déterminant l'étendue de la responsabilité de la société dominante pour les obligations engagées par la filiale à l'égard de ses créanciers. L'absence de notification de cette circonstance provoque la nullité des dispositions du contrat « limitant ou excluant la responsabilité de la société dominante envers ses créanciers " (l'art. $7 \$ 3$ du c.s.c.). La doctrine soulève que les dispositions de l'article 7 du c.s.c. en pratique restent mortes, compte-tenu, entre autres, de l'absence de dispositions prévoyant nettement que le groupe de sociétés doit être considéré, quant au régime fiscal, comme un seul sujet. Ce qui est encore plus important c'est que la disposition susvisée ne constitue pas de fondement juridique matériel susceptible de permettre la création de la responsabilité de la société dominante envers les créanciers de la société dépendante ou d'admettre «la présomption » de l'existence d'une telle responsabilité 5 .

\section{La recherche des fondements d'imputation à la société dominante des comportements propres à la filiale}

Un des outils permettant de " percer le voile corporatif » consiste à imputer à la société dominante certains comportements propres à la société dépendante. Cette mesure consiste à soumettre la filiale aux interdictions ou obligations énumérées se référant directement à la société dominante. Ces sujets sont alors considérés comme une seule personne. On fait ainsi obstacle à l'omission des interdictions (obligations) en se référant à la

3 Les travaux menés dans ce domaine, entre autres dans le cadre de la Commission de codification du croit civil, ont été pour le moment abandonnés.

4 Voir la proposition de la réforme du droit français (cf. F. Terré, Pour une réforme du droit de la responsabilité civile, Dalloz, coll. "Thèmes et commentaires», 2011), publiée en tant que « Rapport Terré sur la Responsabilité civile. - Proposition de textes » sur www.demos.fr (ci-après Rapport Terré).

5 Cf. P. ex. S. Włodyka, Prawo koncernowe (Droit de holding), Cracovie 2003, p. 176. 
société dominante qui, pour atteindre des effets illicites, exploiterait à ses fins sa filiale. Dans la législation polonaise de telles dispositions sont prévues à l'art. $6 \$ 2$ du c.s.c., l’art. $200 \$ 1$ phrase 2 et l'art. $362 \$ 4$ du c.s.c.

Les régulations mentionnées à titre d'exemple se caractérisent pourtant - conformément à la conception de règlementation « ponctuelle » de certains comportements de la société dominante, admise dans la législation polonaise - par une étendue d'application strictement définie. Leur caractère exceptionnel ne permet pas d'adopter, en l'espèce, une interprétation large. Il n'est pas donc bien fondé d'en tirer - en identifiant les comportements de la société dominante à ceux de la société dépendante - une règle stipulant que dans les situations bien définies il est possible d'imputer la responsabilité de la société dominante pour le dommage causé par la société dépendante.

La définition même par la doctrine du phénomène décrit ci-dessus en tant « qu'abus de la forme juridique de la société » incite à envisager la possibilité de recourir à la construction de l'abus de droit définie par la législation polonaise à l'article $5 \mathrm{du}$ Code civil. Il pourrait sembler alors que l'instrument naturel luttant contre l'abus du caractère distinct des sociétés dominante et dépendante, de manière contraire aux règles de la vie en société, soit exactement l'objection portant sur l'abus du droit subjectif.

Néanmoins, la construction de l'abus du droit subjectif adoptée dans la législation polonaise s'avère inefficace pour atteindre la fin considérée dans le cadre de la présente étude. Il en est ainsi puisque, premièrement, le fait que la société dominante exploite la société dépendante en tant que " véhicule » servant à atteindre les objectifs bien déterminés de la société-mère, ne peut pas être considéré comme l'exercice d'un droit subjectif, quel que soit le responsable des effets négatifs de ces actes - la société dépendante elle-même ou bien ses créanciers.

L'absence de responsabilité de la société-mère pour les obligations engagées par la société dépendante constitue indubitablement le fondement de la situation juridique favorable de cette première, sans signifier pourtant qu'elle conduit à la naissance de son droit subjectif, dont l'exercice serait susceptible d'être analysé à la lumière des dispositions de l'article 5 du c.c. La formation de la société ne fait naître aucun rapport juridique entre les associés et les tiers (cocontractants de la société), dans le cadre duquel le droit subjectif existant serait potentiellement l'objet d'un abus exercé par les associés ${ }^{6}$. Deuxièmement, aussi bien la doctrine que la jurisprudence polonaises admettent en principe unanimement que l'instrument d'abus du droit peut servir d'un instrument de défense de la partie défenderesse et non d'un fondement pour formuler une prétention bien déterminée (ouverture d'instance).

6 Ainsi A. Opalski, Prawo (Droit), 485. 


\section{La responsabilité de " percement »}

Par la contestation de la règle d'autonomie, la construction de la responsabilité dite de percement conduit à la suppression de la règle stipulant que l'associé n'est pas responsable pour les obligations de la société.

$\mathrm{Vu}$ les dispositions d'application générale de l’art. $151 \$ 4$ et de l'art. $301 \S 5$ du c.s.c., il manque dans la législation polonaise des fondements juridiques permettant d'éliminer leur application et l'admission de la responsabilité de percement. Quelle que soit la forme de l'influence d'un associé-société dominante exercée sur les affaires de la filiale (exercice d'un contrôle sur la société dépendante, privation de celle-ci de l'actif ou l'absence de l'équipement de celle-ci des moyens nécessaires et ceci en dépit des devoirs dont elle a été chargée).

En principe, la société-mère exerce un contrôle sur la société dépendante dans l'étendue et avec l'intensité lui permettant non seulement de déterminer les objectifs stratégiques de la société mais aussi d'exercer une influence décisive sur ses décisions courantes. A ce titre toutefois, la société dominante n'est pas responsable envers les tiers des effets désavantageux pour la filiale de ses décisions. Il semble en même temps que la création d'une telle responsabilité en tant que principe général de lege ferenda ne serait pas bien fondée. Il en est ainsi puisque ce qui est essentiel pour les groupes de sociétés c'est, entre autres, la centralisation du processus décisionnel dans la société dominante et l'un des principes fondamentaux régissant le droit des sociétés de capitaux est celui de la gérance assurée par la majorité. Son affaiblissement par l'institution de la responsabilité des associés, pour les effets des décisions entreprises à l'égard de la filiale, contredirait des règles fondamentales sur lesquelles est basée la construction de la société jouissant de la personnalité juridique.

La situation où la société ne dispose pas de moyens permettant de réaliser son activité prévue, ce qui entraine (augmente) le risque de sa faillite et ce qui s'en suit, une menace pour les créanciers apparait (augmente), est définie comme "sous-capitalisation » de la société 7 . Cette circonstance ne justifie pourtant pas la formulation d'une objection adressée aux associés de ne pas avoir doté la société de moyens suffisants, puisque le droit polonais ne les soumet pas à une telle obligation. En particulier ils ne sont pas tenus d'adapter le montant du capital social de la société contrôlée ni

7 Cf. P. ex. M. Mieciński, Niedokapitalizowanie spółki z o. o. a wysokość kapitału zakładowego (Sous-capitalisation de la société à responsabilité limitée et le montant du capital social), Przegląd Prawa Handlowego (Revue du Droit Commercial) $1998, n^{\circ} 6$. 
aux dimensions ni au type de l'activité exercée ni de la financer autrement. En principe donc, le fait que la société dominante est bien consciente de l'insuffisance des moyens dont dispose la société dépendante pour accéder à l'activité déterminée, ne suffit pas pour la charger de la responsabilité de percement. Si un tel acte de la société comporte les éléments essentiels d'un délit (où le caractère illicite consiste entre autres dans la violation de l'obligation de loyauté d'un associé à l'égard de la société), il s'agira d'un délit commis envers la filiale et non, de principe, à l'égard de ses créanciers.

De même, la situation où l'on observe « la confusion » des sphères patrimoniales de la société et d'un associé ne justifie pas l'imputation à cet associé de la responsabilité envers les créanciers de la société dépendante. Si une telle "confusion", vu l'inexécution des exigences requises au transfert d'un actif donné du patrimoine de la société au patrimoine de l'associé, n'a pas conduit au résultat prévu, ce composant est accessible aux créanciers de la société. D’autre part, si le transfert au patrimoine de l'associé a été valable, il ne faut analyser qu'une seule question, à savoir s'il a été équivalent, avec le respect des règles relatives à la protection du patrimoine de la société (l'art. 198 et 350 du c.s.c.). La violation est susceptible de constituer un fondement des prétentions formulées par la société à l'égard de l'associé, sans pourtant justifier les prétentions formulées par les créanciers de la filiale qui ont subi un dommage résultant de la diminution de la capacité de la société à régler ses dettes. Par contre, les créanciers peuvent envisager éventuellement le recours à l'instrument de l'action paulienne (l'art. 527 et suiv. du c.c.).

\section{La responsabilité extracontractuelle}

Le seul fondement légal des prétentions éventuelles formulées par les créanciers de la société dépendante envers la société dominante peut être retrouvé dans l'état juridique actuel, dans le régime de responsabilité extracontractuelle $e^{8}$. Le fait qu'un associé dominant agit au préjudice des créanciers de la filiale, peut conduire ces derniers à formuler des demandes d'indemnisation adressées directement à l'encontre de cet associé. En l'espèce, le dommage peut se manifester sous forme d'un préjudice que le créancier de la société dépendante a subi à la suite de l'inexécution par celle-ci de son obligation.

8 Cf. p. ex. A. Karolak, Prawne mechanizmy ochrony spółki córki i jej wierzycieli w strukturze holdingowej (Mécanismes juridiques de la protection de la filiale et de ses créanciers dans le structure de holding), Prawo Spółek (Droit des Sociétés) 2001, n 5 ; A. Opalski, Prawo (Droit), p. 511 et suiv. 
Evidemment il n'est question de telles demandes en indemnisations que si les conditions de responsabilité extracontractuelle sur les principes généraux définies à l'article 415 du c.c. ont été réunies.

Il en résulte donc que, premièrement, les agissements de la société dominante doivent constituer un acte illicite. Pourtant l'associé n'est pas chargé, en vertu d'une obligation générale prévue par la loi, de "veiller aux intérêts » ou de ne pas porter préjudice aux cocontractants de la société dépendante (à ses créanciers potentiels et actuels) ${ }^{9}$. Le fait donc qu'il agit au préjudice de la société dépendante, en violant ses obligations envers elle, ne signifie pas forcément qu'il viole en même temps une obligation quelconque à l'égard des tiers - cocontractants de la société dépendante. Toutefois, dans ce cas-là il peut s'agir de la violation des règles de la vie en société. Selon l'opinion dominante dans la doctrine et la jurisprudence polonaises, cela prouve, en tant que violation d'une règle d'application générale, le caractère illicite du comportement ${ }^{10}$. Une telle perception du caractère illicite justifie que pour illustrer les exemples des comportements illicites de la société dominante, la doctrine cite p.ex. le fait d'avoir donné par un associé dominant à la société dépendante un ordre d'inexécution du contrat liant celle-ci avec un cocontractant ou bien le fait d'avoir conduit à la situation où les obligations de la société dépendante envers ses créanciers ne peuvent pas être réalisées faute de moyens suffisants et finalement on déclare sa faillite. Dans une telle situation, la doctrine soulève également une qualification particulière de la faute de la société dominante ce qui permet de lui imputer la responsabilité envers les créanciers de la société dépendante. On soutient qu'elle doit prendre la forme de animus laedendi c'est-à-dire l'existence de l'intention de causer un dommage aux tiers - créanciers de la filiale, puisque ce n'est que dans ce cas que l'on peut parler de la violation de la règle visant à leur protection ${ }^{11}$.

9 Le caractère illicite d'un acte est une condition requise de l'imputation de la responsabilité non contractuelle - le seul fait d'avoir causé un dommage ne préjuge pas du caractère illicite de l'acte, cf. p. ex. M. Sośniak, Bezprawność zachowania jako przesłanka odpowiedzialności cywilnej za czyny niedozwolone (Le caractère illicite de l'acte en tant que condition de la responsabilité civile des actes illicites), Cracovie 1959, passim ; A. Olejniczak (dans:) Kodeks cywilny, Komentarz (Code civil, Commentaire), dir. A. Kidyba, Varsovie 2010, vol. III, p. 357 ; Z. Banaszczyk (dans :) Kodeks cywilny, Komentarz (Code civil, Commentaire), vol. I, dir. K. Pietrzykowski, Varsovie 2013, p. 1166.

10 Cf. l'art. 5 ph. 2 Rapport Terré : «Un fait est illicite quand il contrevient à une règle de conduite imposée par loi loi ou par le devoir de prudence et de diligence ».

11 Ainsi A. Opalski, Prawo (Droit), p. 512 ; cf. aussi p. ex M. Kaliński, Szkoda na mieniu i jej naprawienie (Dommage survenu sur un bien et sa réparation), Varsovie 2011, p. 110 et suiv. 
Pour pouvoir réfléchir à la possibilité d'imputer à un associé dominant la responsabilité du préjudice causé aux créanciers de la société dépendante, il faut avant tout se référer à l'opinion constamment présente dans la doctrine et la jurisprudence polonaises préconisant l'irrecevabilité d'une demande d'indemnisation au titre d'un préjudice indirect. On soutient alors, que seul est autorisé à demander une réparation d'un préjudice subi celui à l'encontre de qui l'action étant à l'origine du préjudice avait été adressée. On y invoque l'argument selon lequel la législation polonaise manque d'une règle générale conférant la protection de ses intérêts aux personnes directement lésées par un agissement ciblé directement à l'encontre d'un autre sujet. On mentionne aussi souvent la construction dite « d'illicité relative » conduisant à la limitation de l'étendue de l'illégalité au sens large - autrement dit à la limitation subjective de l'étendue de la responsabilité extracontractuelle. Conformément à cette construction, le comportement générateur au sens des dispositions relatives à la responsabilité ex delicto doit avoir été ciblé sur la personne subissant le préjudice. Cela signifie que l'auteur n'est responsable qu'à l'égard des sujets à l'encontre desquels son action a été directement orientée. A ce titre, la victime ayant subi indirectement un préjudice ne peut pas demander la réparation de celui-ci puisque le comportement de l'auteur de l'acte n'a pas porté atteinte à la règle orientée à la protection des intérêts des personnes dont la victime fait partie.

Avec cette interprétation de l'étendue de la responsabilité extracontractuelle de l'auteur de l'acte, en cas de préjudice causé dans le patrimoine de la société dépendante, elle seule en est la victime, et ses créanciers - ayant subi indirectement le dommage - ne peuvent pas réclamer la réparation du préjudice subi. Les partisans de cette opinion soulèvent entre autres que si l'on acceptait la réparation d'un dommage indirect, l'associé dominant serait obligé de réparer le dommage deux fois - une fois à l'égard de la société et l'autre envers ses créanciers. Ils invoquent également les arguments soulevés lors de la discussion portant sur la recevabilité de la formulation par les associés des prétentions au titre des dommages subis par la société à la suite des agissements des tiers et dont l'effet indirect est la diminution de la valeur de leurs droits résultant des parts sociales. Selon l'opinion dominante, en l'espèce la seule victime est la société, tandis que les associés peuvent demander la réparation du préjudice subi par celle-ci en recourant à l'instrument de actio pro socio (cf. l'art. 295 et l'art. 486 du c.s.c.).

Les opinions déclarant l'absence de fondement d'imputer à l'auteur de l'acte la responsabilité "d'un dommage indirect» ne sont pourtant pas bien fondées. Il faut remarquer avant tout que la distinction entre le dommage indirect et direct se fonde souvent dans la législation polonaise, 
étant donné d'ailleurs que celle-ci ne définit pas la notion de dommage indirect et ne s'en sert pas, sur la référence à la position adoptée en la matière par la jurisprudence ou bien sur la signification de la notion de dommage indirect adoptée par un auteur donné ${ }^{12}$. Toutefois la doctrine a formulé de critères différents permettant de distinguer «le dommage indirect ${ }^{13}$. Certains auteurs, comme il a déjà été indiqué, reconnaissent que le sujet qui a subi indirectement un préjudice ne peut pas réclamer la réparation du dommage subi, puisqu'il n'a pas été admis au cercle des destinataires d'une règle en question, ou bien par le dommage indirect ils comprennent un préjudice subi dans la sphère des biens qui ne sont pas directement protégés par la règle juridique donnée.

Dans la doctrine et la jurisprudence on se réfère le plus souvent au critère subjectif simplifié : le dommage indirect est un préjudice subi par un sujet à l'encontre duquel a été orienté l'acte générateur et le dommage indirect est un préjudice subi par un tiers ${ }^{14}$. La victime indirecte est donc celui à l'encontre duquel l'acte générateur n'a pas été orienté et le préjudice subi dans le patrimoine de ce sujet n'a pas été le résultat direct d'un événement ayant été à l'origine du dommage mais il constitue une conséquence secondaire (indirecte) du dommage survenu dans le patrimoine de la victime qui a subi le dommage directement.

Pour soutenir l'argument contestant la possibilité de réclamer la réparation du préjudice subi indirectement on citait également la teneur des dispositions de l'article 446 du c.c. On soulevait alors que l'article

12 Cf. p. ex. M. Safjan (dans:) Kodeks cywilny, Komentarz (Code civil, Commentaire), dir. K. Pietrzykowski, vol. I. Varsovie 2013, p. 1304 ; A. Szpunar, Wynagrodzenie szkody wynikłej wskutek śmierci osoby bliskiej (Réparation du préjudice survenu suite au décès d'un proche), Bydgoszcz 2000, p. 66 et suiv. ; W. Stecki, Problematyka odpowiedzialności za szkodę pośrednią (Problématique de la responsabilité pour un dommage indirect), (dans :) Problemy kodyfikacji prawa cywilnego (studia i rozprawy) (Questions relatives à la codification du droit civil (études et thèses)), Livre commémoratif - hommage au Professeur Zbigniew Radwański, dir. S. Sołtysiński, Poznań 1990, p. 289 ; R. Kasprzyk, Glosa do wyroku Sądu najwyższego z dnia 13 października 1987 (Commentaire à l'arrêt de la Cour Suprême en date du 13 octobre 1987, réf. du dossier IV CR 266/87, OSP 1989, n 7, texte 12.

13 Cf. surtout A. Olejniczak (dans:) System Prawa Prywatnego, Prawo zobowiązań - część ogólna (Régime du droit prix - Obligations - Dispositions générales) v. 6 , dir. Z. Radwański, Varsovie 2009, p. 104 et suiv. ; cf. aussi l'arrêt de la Cour suprême en date du 22 juin 2012, réf. du dossier V CSK 338/11 LEX nº 1228613.

14 Dans la jurisprudence, cf. entre autres les arrêts de la Cour suprême en date du 11 décembre 2008, réf. du dossier IV CSK 349/08, Lex $n^{\circ} 487548$, en date du 28 décembre 1972, réf. du dossier I CR $615 / 72$, OSPiKA, 1974, nº 1, texte 7 , en date du 13 octobre 1987, réf. du dossier IV CR 266/87, OSPiKA, 1988, n 4, texte 96, résolution du 27 avril 2001, réf. du dossier III CZP 5/01, OSP 2003, n 6, texte 74, l'arrêt en date du 12 juin 2002, réf. du dossier III CKN 694/00, OSNC 2003, n 9, texte 124. 
susmentionné de manière exhaustive règle la question de la responsabilité des auteurs pour les dommages à l'égard des victimes ayant subi le préjudice indirectement et qu'il considère le dommage indirect comme une forme légalement définie d'un dommage et non comme l'un des préjudices causés dans le patrimoine de la victime entrant dans la notion générale de dommage. Cette opinion paraît pourtant être mal fondée. C'est plutôt la nature exceptionnelle des prétentions visées à l'article $446 \mathrm{du}$ c.c. qui exigeait une régulation distincte, prévoyant un mode particulier de réparation du préjudice subi par les personnes proches de la victime qui a subi directement le dommage ${ }^{15}$. Ne parait toutefois suffisamment bien fondée la thèse suivant laquelle les dispositions de l'article $446 \mathrm{du}$ c.c. prévoient une dérogation à la règle générale d'irrecevabilité de la demande de protection formulée par la victime qui a subi indirectement le dommage ${ }^{16}$.

L'argument suivant fait référence aux termes de l'article 415 du c.c. selon lesquels celui qui, par sa faute, a causé à autrui un dommage, est tenu de le réparer. On soulève que l'emploi du terme « à autrui » (et non "à quiconque ») signifie l'exclusion de la responsabilité de l'auteur de l'acte à l'égard les victimes ayant subi le dommage indirectement. Toutefois il ne semble pas que cette interprétation, même pour les raisons linguistiques, soit correcte. "Autrui » est opposé à l'auteur et signifie tout simplement " un autre $»^{17}$. La personne qui a subi le dommage peut donc être un «tiers » sujet, alors toute autre personne que celle à l'encontre de laquelle l'acte de l'auteur a été orienté.

Comme il a été évoqué ci-dessus, le caractère illicite signifie un comportement contraire à l'ordre juridique au sens large. Ce comportement est illicite puisqu'il porte atteinte aux obligations ou interdictions et non parce qu'il est orienté vers des sujets bien déterminés ou puisqu'il affecte les intérêts de sujets concrets. Autrement dit, du point de vue des autres sujets, le comportement de l'auteur de l'acte est illicite ou ne l'est pas, indépendamment du fait s'il a porté atteinte aux intérêts de quelqu'un

15 Cf. l'arrêt de la Cour suprême en date du 28 décembre 1972, réf. du dossier I CR 615/72, OSPiKA $1974, n^{\circ} 1$, texte 7.

16 Cf. p. ex. M. Safjan, Problematyka tzw. bezprawności względnej oraz związku przyczynowego na tle odpowiedzialności za niezgodne z prawem akty normatywne (Problématique d'illégalité relative et du lien de causalité sur le fond de la responsabilité pour les actes normatifs illicites) (dans:) Rozprawy prawnicze, Księga pamiątkowa Profesora Maksymiliana Pazdana (Discours juridiques, Livre commémoratif du Professeur Maksymilian Pazdan), dir. L. Ogiegło, W. Popiołek, M. Szpunar, Cracovie 2005, p. 1323.

17 Cf. l'art. 1 al. 1 Rapport Terré « Constitue un délit civil tout dommage illicitement causé à autrui ». 
(a causé un dommage $)^{18}$. Par conséquent la différence dans le traitement des victimes qui ont subi le dommage directement et indirectement n'est pas bien fondée. La réponse à la question si la personne qui a subi un dommage suite à un acte illicite et qualifié fautif peut demander à son auteur la réparation du dommage ne dépend que de la détermination s'il existe un lien de causalité adéquat entre celui-ci et cet acte. L'imputation de la responsabilité est donc déterminée par le critère de causalité défini par la loi. Il en est de même pour ce qui est de l'étendue de cette responsabilité : de l'étendue du dommage indemnisable décide la pertinence des conséquences du comportement générateur. Dans ces circonstances, le préjudice quel qu'il soit (« indirect» ou bien « direct») est indemnisable à condition qu'il soit compris dans l'étendue ainsi définie. C'est ainsi que, de plus en plus souvent, la question de la victime ayant subi «indirectement » un préjudice est analysée par la doctrine ${ }^{19}$ qui s'oppose également à la conception de l'illégalité relative ${ }^{20}$.

Il semble donc que, dans l'état juridique actuel, de principe l'exclusion de la responsabilité de la société dominante pour le dommage causé à un créancier de la filiale ne peut avoir lieu qu'en cas d'absence d'un lien de causalité adéquat entre les agissements de la société dominante et le dommage subi. Si l'acte commis par l'auteur est illicite et qualifié fautif et qu'il a causé (au sens d'un résultat ordinaire) un dommage, chaque victime - ayant subi directement ou indirectement le dommage, a son propre droit à la réparation, la teneur de ce droit revenant à la demande de restituer l'état précédant l'acte préjudiciable.

Il convient donc de retenir la deuxième observation soulevant, que la construction de l'illégalité dite relative sans aucune justification pénètre dans la sphère de liens causaux, propre à l'analyse de la condition d'existence d'un lien de causalité entre l'événement générateur et le dommage causé. Il est également difficile de concilier cette construction avec la

18 Cf. J. M. Kondek, Bezprawność jako przesłanka odpowiedzialności odszkodowawczej (Caractère illicite en tant que condition de la responsabilité non contractuelle), Varsovie 2013, p. 105.

19 Cf. les arrêts de la Cour Suprême en date du 12 juin 2002, réf. du dossier III CKN $694 / 00$, OSNC 2003, n 9, texte 124, en date du 13 octobre 1987, réf. du dossier IV CR 266/87, OSNC 1989, n 9, texte 142 ; résolution en date du 27 avril 2001, réf. du dossier III CZP 5/01, OSNJC de 2001, p. 11 texte 161, l'arrêt en date du 13 juin 2001, réf. du dossier II CKN 507/00, OSP 2002, n 1, texte 3. Dans la doctrine cf. p. ex. M. Safjan, Problematyka (Problématique), p. 1327 ; M. Owczarek, Problem bezprawności względnej w systemie odpowiedzialności deliktowej (Question de l'illégalité relative dans le régime de la responsabilité non contractuelle), Palestra 2004, n 5-6 ; J. M. Kondek, Bezprawność (Caractère illicite) p. 114.

20 Cf. la résolution de la Cour suprême en date du 22 février 2006, réf. du dossier III CZP 8/06, OSNC n $7-8$, texte 123. 
notion d'illégalité au sens des dispositions de l'article 415 du c.c. perçue comme le comportement portant atteinte aux règles de comportement généralement applicables, définies par le bais des obligations et interdictions. Doit être considéré comme illicite aussi bien le comportement orienté à l'encontre d'un bien appartenant à un sujet concret, que celui dont le résultat secondaire est un dommage causé à un tiers.

En reliant les observations ci-dessus à la question analysée dans le cadre de la présente étude, il convient de remarquer que dans la jurisprudence la possibilité d'engager la responsabilité d'un associé de la société à responsabilité limitée au titre d'un dommage causé aux créanciers de la société a été notée dans la décision de la Cour suprême en date du 24.11.2009 ${ }^{21}$. La Cour a indiqué que, bien que l'associé ne soit pas responsable des obligations de la société, il peut être responsable de ses propres comportements qualifiés fautifs, ayant causé un dommage à des tiers. Il est difficile de ne pas partager cette opinion, puisqu'on ne trouve pas d'arguments justifiant la limitation de la responsabilité extracontractuelle là où la loi ne le prévoit pas explicitement.

Il est pourtant évident que c'est aux créanciers de la filiale qu'incombe la charge de démontrer les conditions fondant la responsabilité extracontractuelle de la société dominante. Relever ce défi ne sera pas toujours facile. En premier lieu, il faut démontrer le caractère illicite des agissements de la société dominante. Il peut consister en la contradiction du comportement de la société avec les règles de la vie en société. En particulier, il peut s'agir de l'exercice d'une influence aux intérêts de la filiale conduisant à son insolvabilité2 ${ }^{2}$. Il peut également s'agir de la violation

21 Réf. du dossier V CSK 169/09, LEX n 627248.

22 Il est intéressant de se pencher sur les propositions de la réforme du droit français. Cf. l'art. 1360 « AVANT-PROJET DE REFORME DU DROIT DES OBLIGATIONS (Articles 1101 à 1386 du Code civil) ET DU DROIT DE LA PRESCRIPTION (Articles 2234 à 2281 du Code civil) », préparé sous la direction de P. Catala (dit Avant - projet Catala; publié sur p. ex. www.justice.gouv.fr/art_pix/) se présente comme suit : «En l'absence de lien de préposition, celui qui encadre ou organise l'activité professionnelle d'une autre personne et en tire un avantage économique est responsable des dommages causés par celle-ci dans l'exercice de cette activité. ... De même, est responsable celui qui contrôle l'activité économique ou patrimoniale d'un professionnel en situation de dépendance, bien qu'agissant pour son propre compte, ... Il en est ainsi notamment des sociétés mères pour les dommages causés par leurs filiales... » D'autre part à l'art. 7 phr. 2 et 3 du Rapport Terre nous lirons « Une société ne répond du dommage causé par la société qu'elle contrôle ou sur laquelle elle exerce une influence notable que si, par une participation à un organe de cette société, une instruction, une immixtion ou une abstention dans sa gestion, elle a contribué de manière significative à la réalisation du dommage. Il en va de même lorsqu'une société crée ou utilise une autre société dans son seul intérêt et au détriment d'autrui. » 
de certaines obligations. Toutefois la question qui se pose ne concerne pas la seule obligation d'ordre général de ne pas porter atteinte à autrui, parce que la règle de neminem laedere dans le droit polonais n'est pas appliquée ${ }^{23}$. Le seul fait d'avoir causé un dommage à un tiers ne décide pas de l'obligation de sa réparation, puisqu'il n'est pas en lui-même illicite.

L'imputation de la responsabilité à la société dominante aura lieu surtout dans la situation où les agissements de la société dominante ont été effectués avec l'intention de causer un dommage aux cocontractants, donc dans la situation de faute intentionnelle. Cela ne veut toutefois pas dire que ce soit la seule forme de faute justifiant la responsabilité de la société dominante, puisque dans la législation polonaise il manque de fondements pour subordonner la responsabilité de l'auteur à un degré déterminé de la faute. Si la condition stipulant que le dommage subi indirectement par une personne (créancier de la filiale) constitue un effet « ordinaire» du comportement illicite de son auteur est remplie, la victime peut réclamer la réparation du dommage, même si on ne peut imputer à l'auteur que le moindre degré de la faute.

En ce lieu, on pourrait donc établir une synthèse suivante. Premièrement, les dispositions du droit civil polonais ne prévoient pas la distinction entre le dommage direct et indirect. Deuxièmement, il s'en suit qu'il n'existe pas de régulation susceptible de permettre d'adopter la thèse selon laquelle le dommage "indirect", quelle que soit sa perception, n'est pas indemnisable. Troisièmement, il n'est pas bien fondé de créer une catégorie "d'illégalité relative ", étant donné que doit être considéré comme illicite aussi bien le comportement orienté à l'encontre d'un bien d'un sujet concret, que les agissements dont l'effet secondaire est le dommage causé à un tiers. Quatrièmement, conformément à la règle générale, la victime peut réclamer la réparation du préjudice sous réserve que les conditions requises par la loi soient remplies. Il en résulte que la responsabilité de la société dominante pour le dommage causé à un créancier de la société dépendante peut avoir lieu à condition de démontrer un lien de causalité adéquat entre le comportement illicite et fautif de la société dominante et le dommage constaté.

Il convient également de rappeler les questions formulées dans la doctrine $^{24}$ : si la société dominante a violé ses obligations (celles-ci résultant de la loi, des règles de la vie en société) et qu'elle l'a fait de manière fautive, pourquoi ne serait-elle pas responsable de tous les dommages causés

23 Cf. entre autres M Sośniak, Bezprawność (Caractère illicite) p. 99, A. Szpunar, Czyny niedozwolone w kodeksie cywilnym, Studia Cywilistyczne (Actes illicites dans le code civil, Etudes en droit civil) 1970, vol. XV, p. 51.

24 Ainsi J. M. Kondek, Bezprawność (Caractère illicite), p. 115 
par cet acte, y compris les dommages subis par les créanciers de sa filiale, sous réserve qu'il existe entre cet acte et le dommage un lien de causalité ordinaire? Comment peut-on justifier la limitation de la responsabilité pour cet acte illicite et fautif?

\section{Bibliographie}

Kaliński M., Szkoda na mieniu i jej naprawienie (Dommage survenu sur un bien et sa réparation), Varsovie 2011.

Karolak A., Prawne mechanizmy ochrony spótki córki ijej wierzycieli wstrukturze holdingowej (Mécanismes juridiques de la protection de la filiale et de ses créanciers dans le structure de holding), Prawo Spółek (Droit des Sociétés) 2001, n 5 .

Kasprzyk R., Glosa do wyroku Saqdu najwyższego z dnia 13 października 1987 (Commentaire à l'arrêt de la Cour Suprême en date du 13 octobre 1987), réf. du dossier IV CR 266/87, OSP 1989, $\mathrm{n}^{\circ} 7$, texte 12 .

Kodeks cywilny, Komentarz (Code civil, Commentaire), dir. A. Kidyba, Varsovie 2010, vol. III.

Kodeks cywilny, Komentarz (Code civil, Commentaire), vol. I, dir. K. Pietrzykowski, Varsovie 2013.

Kondek J. M., Bezprawność jako przesłanka odpowiedzialności odszkodowawczej (Caractère illicite en tant que condition de la responsabilité non contractuelle), Varsovie 2013.

Mieciński M., Niedokapitalizowanie spótki z o.o. a wysokość kapitału zakładowego (Sous-capitalisation de la société à responsabilité limitée et le montant du capital social), Przegląd Prawa Handlowego (Revue du Droit Commercial) $1998, n^{\circ} 6$.
Opalski A., Prawo zgrupowań spółek (Le droit de groupement de sociétés), Varsovie 2012.

Owczarek M., Problem bezprawności względnej wsystemie odpowiedzialności deliktowej (Question de l'illégalité relative dans le régime de la responsabilité non contractuelle), Palestra 2004, n 5-6.

Safjan M., Problematyka tzw. bezprawności względnej oraz zwiq̨zku przyczynowego na tle odpowiedzialności za niezgodne z prawem akty normatywne (Problématique d'illégalité relative et du lien de causalité sur le fond de la responsabilité pour les actes normatifs illicites) (dans :) Rozprawy prawnicze, Księga pamiątkowa Profesora Maksymiliana Pazdana (Discours juridiques, Livre commémoratif du Professeur Maksymilian Pazdan), dir. Ogiegło L., Popiołek W., Szpunar M., Cracovie 2005.

Sołtysiński S., Szajkowski A., Szumański A., Szwaja J., Kodeks spółek handlowych, Komentarz (Code des sociétés commerciales, Commentaire), Varsovie 2008 , vol. III.

Sośniak M., Bezprawność zachowania jako przesłanka odpowiedzialności cywilnej za czyny niedozwolone (Le caractère illicite de l'acte en tant que condition de la responsabilité civile des actes illicites), Cracovie 1959. 
Stecki W., Problematyka odpowiedzialności za szkodę pośredniq (Problématique de la responsabilité pour un dommage indirect), (dans :) Problemy kodyfikacji prawa cywilnego (studia i rozprawy) (Questions relatives à la codification du droit civil (études et thèses)), Livre commémoratif - hommage au Professeur Zbigniew Radwański, dir. S. Sołtysiński, Poznan 1990.

System prawa handlowego (Régime du droit commercial). Vol. 2, Prawo spółek handlowych (Droit des sociétés commerciales), Varsovie 2012.
System Prawa Prywatnego, Prawo zobowiq̨zań - część ogólna (Régime du droit privé - Obligations - Dispositions générales) v. 6, dir. Z. Radwański, Varsovie 2009.

Szpunar A., Czyny niedozwolone w kodeksie cywilnym, Studia Cywilistyczne (Actes illicites dans le code civil, Etudes en droit civil) 1970, vol. XV.

Szpunar A., Wynagrodzenie szkody wynikłej wskutek śmierci osoby bliskiej (Réparation du préjudice survenu suite au décès d'un proche), Bydgoszcz 2000.

Włodyka S., Prawo koncernowe (Droit de holding), Cracovie 2003. 